\title{
BMJ Open A pilot randomised controlled trial of personalised care after treatment for prostate cancer (TOPCAT-P): nurse-led holistic-needs assessment and individualised psychoeducational intervention: study protocol
}

\author{
Marian Andrei Stanciu, ${ }^{1}$ Caroline Morris, ${ }^{2}$ Matt Makin, ${ }^{2}$ Eila Watson, ${ }^{3}$ \\ Jenna Bulger, ${ }^{1}$ Richard Evans, ${ }^{1}$ Julia Hiscock, ${ }^{1}$ Zoë Hoare, ${ }^{4}$ \\ Rhiannon Tudor Edwards, ${ }^{5}$ Richard David Neal, ${ }^{1}$ Clare Wilkinson ${ }^{1}$
}

To cite: Stanciu MA, Morris C, Makin M, et al. A pilot randomised controlled trial of personalised care after treatment for prostate cancer (TOPCAT-P): nurse-led holistic-needs assessment and individualised psychoeducational intervention: study protocol. BMJ Open 2015;5:e008470. doi:10.1136/bmjopen-2015008470

\section{- Prepublication history} and additional material is available. To view please visit the journal (http://dx.doi.org/ 10.1136/bmjopen-2015008470).

Received 13 April 2015 Revised 27 May 2015 Accepted 5 June 2015

CrossMark

For numbered affiliations see end of article.

Correspondence to Marian Andrei Stanciu; a.stanciu@bangor.ac.uk

\section{ABSTRACT}

Introduction: Prostate cancer is common and the incidence is increasing, but more men are living longer after diagnosis, and die with their disease rather than of it. Nonetheless, specific and substantial physical, sexual, emotional and mental health problems often lead to a poor quality of life. Urology services increasingly struggle to cope with the demands of follow-up care, and primary care is likely to play the central role in long-term follow-up. The present phase II trial will evaluate the feasibility and acceptability of a nurse-led, person-centred psychoeducational intervention, delivered in community or primary care settings.

Methods and analysis: Prostate cancer survivors diagnosed in the past 9-48 months and currently biochemically stable will be identified from hospital records by their treating clinician. Eligible men would have either completed radical treatment, or would be followed up with prostate specific antigen monitoring and symptom reporting. We will recruit 120 patients who will be randomised to receive either an augmented form of usual care, or an additional nurse-led intervention for a period of 36 weeks. Following the health policy in Wales, the intervention is offered by a key worker, is promoting prudent healthcare and is using a holistic needs assessment. Outcome measures will assess physical symptoms, psychological wellbeing, confidence in managing own health and quality of life. Healthcare service use will be measured over 36 weeks. Feedback interviews with patients and clinicians will further inform the acceptability of the intervention. Recruitment, attrition, questionnaire completion rates and outcome measures variability will be assessed, and results will inform the design of a future phase III trial and accompanying economic evaluation.

Ethics and dissemination: Ethics approval was granted by Bangor University and North Wales REC (13/WA/0291). Results will be reported in peer-reviewed

\section{Strengths and limitations of this study}

- The intervention is designed in line with new Welsh health policy by promoting prudent healthcare principles and offering a key worker for each cancer survivor.

- The holistic needs assessment uses novel and comprehensive instruments bridging research and hospital best practice, which will be shared with patients, primary and secondary care.

- The study adopts an augmented form of usual care, in line with ongoing developments of the care system in the recruitment area.

- The intervention is offered to stable survivors, irrespective of risk-stratification, or self-reported level of need, for an accurate assessment of its overall effectiveness.

- Recruitment area covers rural as well as urban regions, with a wide mix of socioeconomic strata.

publications, at scientific conferences, and directly through national cancer and primary care networks. Trial registration number: ISRCTN 34516019.

\section{INTRODUCTION}

Prostate cancer is the most common cancer for men in the UK (second worldwide), and many survivors experience long-lasting physical and psychological needs. Over the past 20 years, in the UK, incidence rates have doubled, but mortality rates have dropped by a quarter. ${ }^{1}{ }^{2}$ Common physical symptoms are related to sexual function, urinary incontinence, ${ }^{3}$ bowel symptoms, ${ }^{4}$ hot flushes and the risk of bone fracture. ${ }^{5}$ The management of chronic comorbid conditions (eg, cardiovascular disease and cerebrovascular disease, 
hypertension, diabetes) often further increases the level of need, and about two-thirds of patients with prostate cancer are expected to have at least one major comorbidity. ${ }^{6-8}$ Psychological distress is also significant, and most prostate cancer survivors require prompt information about treatment outcomes and its impact on daily living. ${ }^{9}$ The diagnosis and treatment toxicities also affect the patients' immediate families, ${ }^{10}$ particularly through psychological distress related to anxiety, depression $^{11} 12$ and psychosexual problems. ${ }^{13}$ Thus, the assessment and management of the adverse treatment effects, related psychosocial needs (also affecting their partners) and the impact on the management of other comorbid conditions is, for many patients, complex and prolonged.

\section{Current usual care and evidence base}

Patients with prostate cancer are normally followed up in out-patient clinics in hospital for up to 5 years, to monitor and manage the risk of recurrence, and the physical symptoms following treatment. ${ }^{14}$ However, current practice is not underpinned by robust evidence, and is notoriously variable between hospitals. ${ }^{15}$ In the absence of reliable empirical evidence, the National Institute for Health and Care Excellence (NICE) guidelines recommend that unless significant treatment complications develop, after 2 years, their follow-up care should take place out of hospital. ${ }^{16}$ However, recommendations on the type of follow-up to be undertaken are notably missing from the guidelines.

In the last decade and a half, attempts have been made to address the lack of empirical evidence regarding the efficacy and cost-effectiveness of prostate cancer follow-up. Early initiatives showed that by involving primary and community care, the utilisation of specialist care may be reduced, especially for the more elderly patients. ${ }^{17}$ Also, patients perceive they receive more care from the general practitioner (GP),${ }^{18}$ while their quality of life remains similar between hospital and primary care follow-up. However, notable concerns were reported about the continuity of care, the miscommunication between hospitals and GPs and the integration of prostate specific antigen (PSA) testing. A number of hospitalbased alternatives have been proposed, such as hospital group clinics, ${ }^{19}$ nurse face-to-face and telephone clinics $^{20-22}$ and e-health technology based follow-up. ${ }^{23-25}$ Such approaches fail to address the issues about the capacity and scope of specialist secondary care teams, which may struggle to offer, assess and manage a holistic range of physical, psychosocial and educational needs of patients. Recently, improvements in e-health platforms facilitating the communication between hospital and primary care, especially surrounding the safe monitoring of PSA levels and cancer recurrence, have revived efforts to consider a primary-care-led model of follow-up. ${ }^{26}$

Nurse-led interventions have been consistently shown to be effective in a range of diseases, from diabetes and depression, ${ }^{27} 28$ to various cancers, ${ }^{29}$ and, more specifically, when interventions were administered in primary care settings. ${ }^{30}$ There is sufficient literature showing the gaps in care to argue for a more intensive approach initially, ${ }^{31}$ and most emerging models include a nurse-led assessment of needs. There is evidence that increasing the participation of patients with cancer in their own care can reduce their psychosocial and information needs. ${ }^{32}$ Self-management is now accepted as a potential solution for the complex needs of prostate cancer survivors, ${ }^{33}$ but conclusive evidence is still needed regarding the design and delivery of such interventions.

\section{Person-centred and prudent healthcare for prostate cancer survivors}

Despite a tradition of predominantly disease-centred follow-up, the person-centred approach features highly in the UK health policy agenda. The 2004 NICE guidelines ${ }^{34}$ recognised the complex needs of cancer survivors, and the Cancer Reform Strategy ${ }^{35}$ set out to understand and address them. In Wales, the government's Together for Health-Cancer Delivery Plan ${ }^{36}$ directed Health Boards to assign a Key Worker to assess and record the clinical and non-clinical needs of cancer survivors in a personalised care plan, and to ensure care is coordinated between hospital and community. The policy highlighted the need for new multidisciplinary models of follow-up to be developed and evaluated. Moreover, the Bevan Commission ${ }^{37}$ recommended the application of prudent healthcare principles, such as: (1) offering early interventions, (2) promoting selfmanagement and the coproduction of healthcare, (3) involving community assets in order to reduce the level of unmet need, (4) removing unnecessary processes (especially the duplication of support services), and (5) adopting services that achieve similar or better patient outcomes, while using less expensive human and technical resources. Thus, for prostate cancer follow-up, the government health policy directs towards holistic and person-centred care, delivered safely and at the earliest opportunity, outside of hospital, with the aim of empowering patients to take an active role in managing and improving their health.

The present trial (TOPCAT-P) is directly addressing the growing capacity challenges facing hospital based services in the UK, by engaging primary and community care soon after the end of prostate cancer treatment. The pilot trial, PROSPECTIV, ${ }^{14}{ }^{38}$ served as the basis for the development of the present work. TOPCAT-P is expanding the personalised nurse-led intervention being piloted in PROSPECTIV in three significant areas: (1) the intervention (including the holistic needs assessment) is being offered irrespective of the patient-reported level of need; (2) the care planning documentation and sharing are updated in response to on-going changes to policy and practice; (3) all participants in the intervention and control arms will receive Macmillan written materials as part of usual practice. The manualised nurse-led 
psychoeducational intervention includes an exploratory and person-centred holistic needs assessment, promotes self-management of symptoms, is delivered out-ofhospital and includes patients' partners, carers, or close family members, where necessary. The aim of the current pilot trial is to evaluate the feasibility and acceptability of the intervention, addressing the wider group of cancer survivors, using the novel holistic needs assessment and care planning tools.

\section{METHODS AND ANALYSIS}

\section{Trial design}

TOPCAT-P is a randomised two-arm parallel-group phase II external feasibility trial, comparing the effectiveness and cost-effectiveness of a personalised, nurse-led, psychoeducational intervention versus the augmented version of usual care beginning to be delivered in North Wales. The present trial follows the new Medical Research Council (MRC) guidelines for the development of complex interventions ${ }^{39}$ by investigating the feasibility and acceptability of the intervention, the novel holistic needs assessment instruments, and enhanced information documenting and sharing procedure. This will be used to inform the design of a phase III trial, which will assess the effectiveness and cost-effectiveness of the intervention.

\section{Participant recruitment and consent Inclusion criteria}

The Urology Clinical Nurse Specialist will identify biochemically stable incident patients with prostate cancer, 9-48 months postdiagnosis, from the multidisciplinary team (MDT) records in the Wrexham Maelor Hospital. They would have either received radical curative treatment (surgery, radiotherapy, or hormone therapy), or be followed up with PSA monitoring and symptom reporting, but deemed unlikely to receive curative treatment (watchful waiting). Notably, patients currently followed up in the hospital or in the community will be invited to participate in the study.

\section{Exclusion criteria}

The study will exclude men suitable for curative treatment, but who choose to be monitored until proof of further progression (active surveillance). Also, palliative patients who are in the terminal stage of their disease or who lack the capacity to consent (as assessed by the referring clinician) will not be included.

\section{Sample size}

We intend to invite 300 patients to take part in the pilot trial and estimate a recruitment rate of approximately $40 \%$. This will allow the recruitment of 120 participants (60 per trial arm-optimum for the randomisation procedure described below). ${ }^{40} \mathrm{~A}$ maximum attrition rate of $50 \%$ will ensure at least 30 patients per arm will complete the trial. This will provide a satisfactory number of participants for estimating the variation within the sample (ie, the SD of the outcome measures), in order to inform the power calculation for a future phase III trial, which would be powered to detect clinically relevant changes in prostate-related health and costeffectiveness.

\section{Randomisation}

Participants will be randomised individually to one of the two arms of the trial (usual care or nurse-led intervention), on a 1:1 basis and stratifying for age (65 or under, 66-72, 73-80, over 80), according to the Cancer Incidence Report 2007-2011. ${ }^{41}$ The concealed allocation procedure will use a secure, off-site electronic system managed by the North Wales Organisation for Randomised Trials in Health (NWORTH) - a UKCRC fully registered trials unit. The system uses a sequential dynamic adaptive randomisation algorithm, ${ }^{40}$ tuned to balance within stratification levels and overall, while maintaining an acceptable level of unpredictability.

\section{Augmented usual care}

Patients in both arms of the study will continue to receive the usual care delivered outside of the trial, including any follow-up appointments (at the hospital or general practice). To account for the variable patterns of follow-up care, all contacts with healthcare professionals will be recorded in bespoke health service-use diaries (client service receipt inventory (CSRI)). To reflect the changes to usual care being implemented in Wales, all patients will be offered, in person, after providing informed consent (see online supplementary appendices 1 and 2), a Macmillan Organiser ${ }^{42}$ to help selfrecord and monitor any physical and psychological symptoms, as well as the results of relevant medical tests and medication taken. All patients will also be signposted to contact the local Macmillan information centre for information and advice regarding any cancerrelated concerns, as well as to contact their GP or hospital team, if necessary, for appropriate medical support.

\section{Intervention}

Supplementary to augmented usual care, patients in the intervention arm will be offered an initial appointment with the research nurse for a holistic needs assessment, and tailored follow-up appointments, as appropriate. Before the start of the intervention, the research nurse will complete the 2-day course, 'The detection of psychological distress in patients with cancer', needed for National Health Service (NHS) staff to qualify at level 2 of the 4-tier model of Psychological Support. ${ }^{34} 43$ Additionally, through the Macmillan network, the nurse will complete three training modules routinely recommended for clinical staff delivering holistic needs assessments: 'Maguire Advanced Communication Skills' training, ${ }^{44}$ 'Motivational Interviewing' and '10-min CBT' ${ }^{45} 46$ The intervention will make use of dynamic personal care plans, and encourage self-management 
(empowering men to help themselves). Distinctly, the research nurse will use a comprehensive holistic needs assessment tool and care plan ${ }^{47}$-specifically exploring physical, emotional, spiritual, lifestyle and family aspects of cancer survivorship, together with an additional bespoke instrument developed in secondary care to monitor physical symptoms (H Nikkhouy-Toussi. Consultant urological surgeon. Personal communication, 16 September 2013). Following the assessment, the nurse will provide individualised information, advice and support tailored to each patient, in order to help men improve their symptoms or cope better with symptoms they cannot improve. Patient referral to GP or secondary care and signposting to community or third sector support services will be made as appropriate. The holistic needs assessment will be documented and shared with patients and, following consent, with their GP. If acute physical symptoms are identified or if disease recurrence is suspected, these will be communicated directly to the secondary care team and GPs. All referrals to tertiary services will be documented in the secondary care cancer network information system (CaNISC) to be available to Oncology teams and facilitate seamless care between healthcare providers. ${ }^{48}$
The initial holistic needs assessment

The first appointment will be in person and will take place out of hospital, in the patient's own primary care setting (by agreement with the general practice), at the local community hospital, or, alternatively, in a dedicated space at the research unit. Housebound patients will be offered home visits. The needs assessment will explore a comprehensive range of symptoms and concerns (see table 1). The nurse will encourage patients to consider all the aspects of survivorship, and will specifically explore symptoms and concerns beyond the formalised checklist.

The delivery of the intervention is based on the novel needs assessment instruments and care plan. ${ }^{47}{ }^{49}$ Following the assessment, a range of person-tailored and symptom-specific management strategies will be taught. Physical and psychological needs will not be treated separately, but in relation to each other. ${ }^{50}$ Thus, physical management techniques (eg, pelvic floor exercises, double-void technique) will be taught in the context of established cognitive-behaviour therapy techniques such as self-monitoring, guided-discovery, life-style adjustment and cognitive re-appraisal. ${ }^{51}$ The nurse will invite patients to examine their lifestyle prior to their prostate cancer diagnosis, identify how their thoughts, ideas,

Table 1 Summary of holistic needs assessment

\begin{tabular}{|c|c|c|}
\hline Categories of need & Symptom & Summary of key assessment points \\
\hline \multirow[t]{9}{*}{ 1. Physical symptoms } & 1. Pain & Type of pain, duration and level of pain \\
\hline & 2. Breathing problems & Relevant comorbidities \\
\hline & 3. Appetite & $\begin{array}{l}\text { Appetite levels, weight loss, soreness to the mouth, difficulties with } \\
\text { digestion, symptoms of nausea or vomiting }\end{array}$ \\
\hline & 4. Urinary function & $\begin{array}{l}\text { Lower urinary tract symptoms, bleeding, incontinence concerns, impact on } \\
\text { everyday life (including psychological impact) }\end{array}$ \\
\hline & 5. Bowel function & $\begin{array}{l}\text { Loose stools, bleeding or incontinence, impact on everyday life (including } \\
\text { emotional aspects) }\end{array}$ \\
\hline & 6. Mobility & $\begin{array}{l}\text { Limitations to mobility, relations to fatigue, impact on mood, general } \\
\text { well-being and energy levels }\end{array}$ \\
\hline & 7. Fatigue & $\begin{array}{l}\text { Dietary intake, impact on mood, enjoyment of daily activities, quality of } \\
\text { sleep, background stressors, fears or anxieties, relaxation therapies, } \\
\text { organisation of daily activities }\end{array}$ \\
\hline & 8. Sexual function & $\begin{array}{l}\text { Erectile dysfunction, loss of libido, impact on relationship with partner, } \\
\text { patients' and partner's feelings, and anxieties }\end{array}$ \\
\hline & 9. Hot flushes & Emotional impact, participation in social activities, relations with others \\
\hline \multirow[t]{8}{*}{$\begin{array}{l}\text { 2. Emotional concerns, } \\
\text { anxieties }\end{array}$} & 1. Depression & $\begin{array}{l}\text { Low mood, loss of interest in everyday activities, depressive thoughts, } \\
\text { behaviour changes, isolation, social relations, utility of mood record }\end{array}$ \\
\hline & 2. Anger & $\begin{array}{l}\text { Anger towards diagnosis, guilt at causing stress to partner or family, strain } \\
\text { on relationships }\end{array}$ \\
\hline & $\begin{array}{l}\text { 3. Fear of disease } \\
\text { recurrence }\end{array}$ & Lifestyle before diagnosis, hobbies, regular PSA monitoring \\
\hline & $\begin{array}{l}\text { 4. Altered body } \\
\text { image/sexuality }\end{array}$ & $\begin{array}{l}\text { Weight gain/loss, breast swelling, impact on mood and sexuality, } \\
\text { behavioural changes, healthy nutrition, regular exercise }\end{array}$ \\
\hline & 5. Spirituality & Loss of faith, meaning of life after diagnosis \\
\hline & 6. Financial concerns & $\begin{array}{l}\text { Loss of finance, insecurities about future earnings/costs, inability to afford } \\
\text { past hobbies, financial support }\end{array}$ \\
\hline & 7. Lifestyle changes & Travel insurance, planning of daily journeys, self-monitoring of symptoms \\
\hline & $\begin{array}{l}\text { 8. Memory and } \\
\text { attention }\end{array}$ & Increased overall stress, general self-confidence, change in sleep patterns \\
\hline
\end{tabular}


feelings, attitudes and behaviours affect their day-to-day life, and to reflect on the impact this is having on their life. For patients who have fully adjusted to survivorship, the process is expected to be relatively quick and seamless. However, patients who experience any level of unmet need will first benefit from the guided selfreflection. ${ }^{52}$ Second, where action is necessary to address individual symptoms, patients will be offered specific and personalised advice. Supported by the nurse, patients will consider which aims and strategies are attainable and relevant for their circumstances. A plan will be devised together with the nurse to accomplish these goals, and will be documented in the personalised care plan. A copy of the initial holistic needs assessment summary and the complete personalised plan of care will be given to patients, and, with their consent, will be sent to their GP for information and long-term management. A covering letter will explain to GPs the context of the care plan, the scope and duration of the intervention, and will provide a point of contact for any related queries. Where the level of support and complexity of need will exceed the capability of the current intervention, the nurse will specifically refer patients to their GP or for specialist support, as appropriate.

\section{The follow-up sessions}

By agreement with patients, the nurse will arrange follow-up appointments to monitor the progress of the self-management strategies advised during the initial assessment. The progress made and any related patient concerns will be documented in the patient's plan of care, and again shared with patients and their GP, as before. The accompanying covering letter will inform GPs of the remaining support available from the intervention, and the outstanding patient needs and concerns. Patients will also be given the opportunity to request follow-up sessions at any point during the intervention by contacting the nurse by telephone. As above, follow-up appointments will take place in general practice, community hospital, the research unit, or at the patient's home, for housebound patients. These appointments will be in addition to any referrals to support outside the intervention. We anticipate men will need on average 1-2 follow-up sessions, but their number will not be limited. The frequency, setting and content of these sessions will be recorded by the nurse for the process evaluation.

\section{Outcome measures}

As a phase II trial, the primary measures of interest are patient recruitment, attrition and response rate for questionnaires. To capture the intervention outcome, a battery of established patient reported measures will be used to assess changes in the physical symptoms (Expanded Prostate Cancer Index Composite, EPIC-26), ${ }^{53}$ psychological well-being (Hospital Anxiety and Depression Scale; HADS),${ }^{54}$ confidence in managing own health,${ }^{55}$ medical and support needs (Supportive Care Needs Surveysimplified response format), ${ }^{56} 57$ and general health and quality of life (EuroQoL EQ-5D-5L), ${ }^{58}$ along with a bespoke questionnaire assessing patients' satisfaction with the healthcare services. To reduce participant burden, the questionnaires have been collated in a single booklet. All questionnaires will be self-completed by patients. The researcher recruiting the patients will offer the baseline measures to all patients after consent, and prior to randomisation. The researcher will remain blind to the randomisation results until the end of recruitment. Subsequent questionnaires will be sent by post to be completed by patients in both arms and similarly returned to the research team by post (see table 2). The ongoing use of health and social care services during the intervention will be collected at 12, 24 and 36 weeks, using a purposebuilt diary. The questionnaire documents the frequency and types of contacts with primary and secondary healthcare services, social services and voluntary sector services. The diary will include information about: the number of times the patient had to see a doctor, nurse, or other healthcare professional in relation to his prostate cancerrelated symptoms; the special medication, aids and adaptations prescribed to patients to help with their prostate cancer-related symptoms; and the number of days patients felt too unwell to participate in their normal activities due to prostate cancer-related symptoms. Moreover, relevant medical history data (eg, cancer diagnosis, stage, treatment type, chronic and acute comorbid conditions, etc) will be collected from GP-and hospital-held records with patients' consent.

A subsample of patients in the intervention group $(\mathrm{N}=32)$, the research nurse and secondary and primary care clinicians $(\mathrm{N}=10)$, will be invited to take part in individual feedback interviews at the end of the trial. Patients will be selected through purposive sampling to include all types of treatment (surgery, radiotherapy, hormone therapy, watchful waiting), cancer stage (localised, locally advanced/advanced), and represent a balanced median split for age and level of need. Clinicians will be selected from those who had the largest number of patients in trial. A researcher not involved in the intervention delivery will conduct the interviews face-to-face, or alternatively by telephone. The interviews will be semistructured and investigate the patients' experience of the intervention, the perceived benefits and missed opportunities of the trial, and possible effects beyond those captured in the proposed outcome measures (both for patients and healthcare services/clinicians).

\section{Data safety and monitoring}

The study procedure and intervention were assessed to present only low impact risks for patient safety, with a low probability. Thus, an independent data monitoring group will not be needed, and interim analyses will not be conducted. However, the intervention will be continuously monitored for safety by the research team, 
Table 2 Timeline of intervention delivery and outcome measures

\begin{tabular}{|c|c|c|c|c|c|c|c|}
\hline \multicolumn{4}{|c|}{ Augmented usual care } & \multicolumn{4}{|c|}{ Nurse-led intervention } \\
\hline T0 & T1 & T2 & T3 & T0 & T1 & T2 & T3 \\
\hline Consent & 12 weeks & 24 weeks & 36 weeks & Consent & 12 weeks & 24 weeks & 36 weeks \\
\hline
\end{tabular}

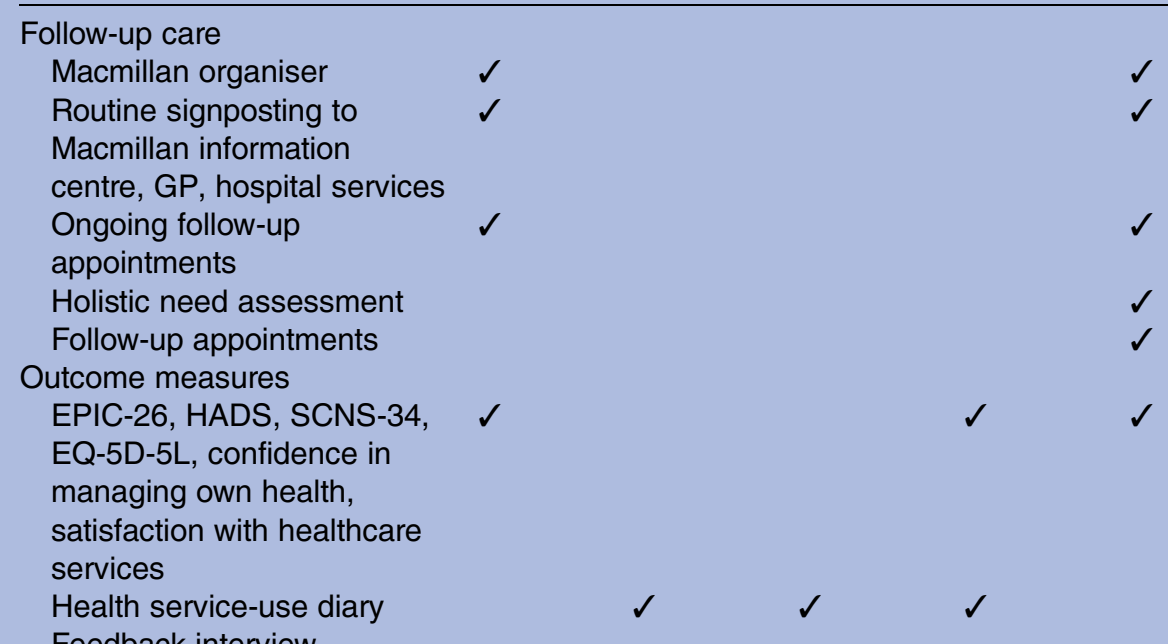

Ongoing follow-up

appointments

Holistic need assessment

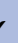

utcome measures

EPIC-26, HADS, SCNS-34

EQ-5D-5L, confidence in

managing own health

satisfaction with healthcare

Health service-use diary

Feedback interview

EPIC-26, Expanded Prostate Cancer Index Composite; GP, general practitioner; HADS, Hospital Anxiety and Depression Scale;

SCNS, Supportive Care Needs Survey.

with direct input from the patients' general practice and referring secondary care clinicians. All process and safety monitoring records will be maintained in accordance with national and local research governance regulations. All adverse events and serious adverse events will be recorded, and followed up for the duration of the study or until resolution. Assessment of adverse events will be performed by the clinical lead of the research team. All serious adverse events will be graded and reported to the sponsor, funder, and the ethics and research governance committees.

\section{Data management}

All data will be collected on paper questionnaires, which will be stored, linked and entered electronically in an anonymised format. Routine data checks will be performed at two time points: (1) when the questionnaires are received from patients, and (2) when the data are entered into a secure electronic data capture system (MACRO, V.4.2.4, InferMed Limited), hosted and managed by the clinical trials unit (NWORTH). Electronic data will be audited on an ongoing basis by two independent auditors, and outcomes will inform the remaining data collection and entry. All data queries will be managed directly by a single Data Manager, and the complete audit trail will be recorded electronically in MACRO. At the end of the trial, all paper questionnaires and electronic data will be archived securely and stored for 5 years, after which they will be confidentially destroyed.

\section{Data analysis}

Feasibility metrics (eg, recruitment and retention rates, clinical characteristics, randomisation, duration of the intervention, etc) will be analysed first, together with adherence outcomes (patient acceptance and adherence to the intervention). Medical history data will be assessed for completeness in conjunction with the outcome measures. The semistructured interviews will be analysed using the matrix based thematic Framework approach, which facilitates analysis both by case and theme. ${ }^{59} 60$

A preliminary analysis of the intervention outcomes will be carried out, following an intention-to-treat approach. Point and 95\% CI estimates will be calculated for the changes in prostate-specific symptoms, quality of life, psychological well-being, self-confidence in managing own health, and ongoing medical and support needs between the two groups. Results will be used to estimate SDs and effect sizes to help inform a sample size calculation for a future phase III randomised control trial (RCT) - if feasibility and acceptability are confirmed.

The analysis will also address the health economics of the intervention. The benefit measurement will use both generic health-related quality of life (EQ-5D-5L) and prostate cancer specific quality of life measures. The analysis will take a societal perspective, given the broad impact on the NHS (both primary and secondary care), the patients, their families and the third sector. In line with established guidelines for economic evaluation of complex interventions, ${ }^{61}$ the costing analysis will use the national unit costs. ${ }^{62}$ The outcome of the preliminary economic analysis estimates will serve to develop the protocol for a full primary cost utility analysis, with a secondary cost-consequence analysis, in a future phase III RCT.

\section{DISCUSSION}

The TOPCAT-P trial proposes a novel model of care for prostate cancer survivors, in line with recent NICE 
guidelines, local government health policy and charity sector initiatives in Wales, to offer a holistic and personalised care delivered in primary and community care settings. These changes to presently hospital-based models of care come in response to increased levels of patient unmet need, raising numbers of prostate cancer survivors with continuing upward estimates, and unavoidable logistical and financial pressures on secondary care teams.

The present trial aims to evaluate the feasibility and acceptability of the intervention, addressing the wider group of cancer survivors, using the novel holistic needs assessment and care planning tools, in the context of care in Wales. The results will inform the design of a definitive stage III trial, for this model of prostate cancer follow-up. A phase III; RCT would be deemed feasible if: (1) a minimum of $25 \%$ of the clinically eligible patients, who will be invited to take part in the trial are recruited, (2) the attrition rate during the trial is no greater than $20 \%$, and (3) the outcome measures completion rate for the active participants (ie, those who have not withdrawn, died or been lost to follow-up) is above $66 \%$. All the feasibility metrics will be calculated using 95\% CIs. Moreover, the patients' and clinicians' feedback will be used to assess the acceptability of the intervention and shape its future administration as well as the overall communication with participants and healthcare professionals. The recruitment, attrition and questionnaire completion rates, together with the SD of the main intervention outcomes will inform the estimation of the sample size for a future phase III trial. The time needed to collect and analyse the data will be used to determine the optimum timings of each activity, and the overall duration of the trial.

The nurse-led intervention piloted in TOPCAT-P is based on a similar trial conducted in England (PROSPECTIV), ${ }^{38}$ but is significantly different in three methodological areas, which will extend the knowledge gained from PROSPECTIV, and assess the feasibility and acceptability of the intervention in a different area, and in different settings. First, the intervention is offered to stable prostate cancer survivors irrespective of their self-reported level of need. Second, the holistic needs assessment, care planning and information sharing documentation is based on novel instruments currently being considered for routine clinical use in the TOPCAT-P recruitment area. Third, the definition of usual care is updated in response to on-going changes to practice in Wales, including new routine third sector improvements, which will provide a contextualised assessment of the intervention's effects. However, similarly to PROSPECTIV, the intervention is nurse-led, based on a psychoeducational framework, promotes self-management of symptoms, is delivered in the community and includes patients' close social group (eg, partner, family, carers), where this is relevant and helpful for the patient.
Author affiliations

${ }^{1}$ North Wales Centre for Primary Care Research, Bangor University, Wrexham, UK

${ }^{2}$ Betsi Cadwaladr University Health Board, Wrexham, UK

${ }^{3}$ Department of Clinical Health Care, Oxford Brookes University, Oxford, UK

${ }^{4}$ North Wales Organisation for Randomised Trials in Health, Bangor

University, Bangor, UK

${ }^{5}$ Centre for Health Economics and Medicines Evaluation, Bangor University, Bangor, UK

Acknowledgements The authors would like to thank our many collaborators who have contributed to this study. The trial is based on the collaborative work of the PROSPECTIV team in Oxford, led by Professor Eila Watson. Marie Burrows conducted a part of the early developmental work. Sandie Jones, Advanced Nurse Practitioner (ANP), screened and invited patients into the trial. Mr Hamid Nikkhouy-Toussi, Consultant Urologist, advised on secondary care prostate cancer follow-up and provided the forms he previously developed in the hospital to monitor patients' progress post-treatment. Guidance on data management has been provided by Dr David Hunnisett from the North Wales Organisation for Randomised Trials in Health (NWORTH). The authors are grateful to all the trial participants, general practitioners (GPs), practice managers, hospital specialists and research interns involved in this trial.

Contributors CW, MM, EW, RE, JB and RDN coordinated the design and funding award. MAS finalised the design of the recruitment, and data collection processes and instruments, managed the ethical and governance approval process, led the implementation of the study and the writing of the manuscript. CM contributed to the development of the intervention and the symptom screening and assessment instruments, the screening of patients, delivered the intervention and collected the medical data from general practice. CW, RDN and MM provided clinical expertise. ZH provided expertise regarding the design, sampling, randomisation and statistical analysis plan for the quantitative measures. JH provided expertise regarding the design, sampling and analysis plan of the feedback interviews. RTE provided expertise on the economic evaluation and health economic instruments, data collection and analysis. All authors had a significant contribution to the manuscript and approved the final version.

Funding This trial is fully funded by Macmillan Cancer Support (grant ID: 4237574).

Competing interests MAS, CW, RE, JH, ZH, RTE and RDN are employed by Bangor University. MM and CM are employed by Betsi Cadwaladr University Health Board. RDN's and CW's posts are part funded by Public Health Wales. RDN's post is also part funded by Betsi Cadwaladr University Health Board. RDN currently holds research grants from NIHR, NISCHR, Cancer Research UK, Cancer Research Wales, HTA and Tenovus Cancer Care. CW currently holds research grants from Cancer Research UK, Macmillan Cancer Support, NISCHR, Tenovus Cancer Care and HTA.

\section{Patient consent Obtained.}

Ethics approval Ethics approval has been granted by Bangor University (HMSAEC, School of Healthcare Sciences), and by the North Wales Research Ethics Committee (Central and East) and the Betsi Cadwaladr University Health Board Research and Development Internal Review Panel (13/WA/0291). All protocol amendments will be communicated to the Ethics Committee (substantial amendments) and to the $\mathrm{R}$ and $\mathrm{D}$ review panel (minor and substantial amendments) for approval, according to the research governance regulations in Wales, UK. With the funder's consent, results will be reported in peer-reviewed journals, presented at scientific conferences, and disseminated directly to policymakers, and more widely through national cancer and primary care networks. All participants in the trial will be offered a summary of the study's outcomes.

Provenance and peer review Not commissioned; peer reviewed for ethical and funding approval prior to submission.

Data sharing statement No additional data are available.

Open Access This is an Open Access article distributed in accordance with the Creative Commons Attribution Non Commercial (CC BY-NC 4.0) license, 
which permits others to distribute, remix, adapt, build upon this work noncommercially, and license their derivative works on different terms, provided the original work is properly cited and the use is non-commercial. See: http:// creativecommons.org/licenses/by-nc/4.0/

\section{REFERENCES}

1. Cancer Statistics. Cancer of the prostate. Information Services Division Scotland (ISD Scotland). http://www.isdscotland.org/HealthTopics/Cancer/Cancer-Statistics/Male-Genital-Organs/\#prostate (accessed 8 Jan 2015).

2. Cancer survival in England: Patients diagnosed 2005-2009 and followed up to 2010. London: Office for National Statistics (ONS) 2011. http://www.ons.gov.uk/ons/publications/re-reference-tables. html?edition=tcm\%3A77-239726 (accessed 8 Jan 2015).

3. Ream E, Quennell A, Fincham L, et al. Supportive care needs of men living with prostate cancer in England: a survey. $\mathrm{Br} J$ Cancer 2008;98:1903-9.

4. Smith D, King M, Egger S, et al. Quality of life three years after diagnosis of localised prostate cancer: population based cohort study. BMJ 2009;339:b4817

5. Shahinian VB, Kuo Y-F, Freeman JL, et al. Risk of rracture after androgen deprivation for prostate cancer. $N$ Engl J Med 2005;352:154-64

6. Armes L, Crowe M, Colbourne L, et al. Patients' supportive care needs beyond the end of cancer treatment: a prospective, longitudinal survey. 2009. J Clin Oncol 2008;27:6172-9.

7. Crawford ED, Grubb R III, Black A, et al. Comorbidity and mortality results from a randomized prostate cancer screening trial. J Clin Oncol 2011;29:355-61.

8. Daskivich TJ, Fan $\mathrm{KH}$, Koyama $\mathrm{T}$, et al. Effect of age, tumor risk and comorbidity on competing risks for survival in a US populationbased cohort of men with prostate cancer. Ann Intern Med 2013:158:709-17.

9. Cockle-Hearne J, Charnay-Sonnek F, Denis L, et al. The impact of supportive nursing care on the needs of men with prostate cancer: a study across seven European countries. Br J Cancer 2013;109:2121-30

10. Harden J, Sanda MG, Wei JT, et al. Survivorship after prostate cancer treatment: spouses' quality of life at 36 months. Oncol Nurs Forum 2013;40:567-73.

11. Northouse LL, Mood DW, Montie JE, et al. Living with prostate cancer: patients' and spouses' psychosocial status and quality of life. J Clin Oncol 2007;25:4171-7.

12. Segrin C, Badger TA, Harrington J. Interdependent psychological quality of life in dyads adjusting to prostate cancer. Health Psychol 2012;31:70-9.

13. Tanner T, Galbraith M, Hays L. From a woman's perspective: life as a partner of a prostate cancer survivor. J Midwifery Womens Health 2011;56:154-60.

14. Watson E, Shinkins B, Frith E, et al. Symptoms, unmet needs, psychological well-being and health status in prostate cancer survivors: implications for redesigning follow-up. BJU Int Published Online First: 23 May 2015. doi:10.1111/bju.13122

15. Bulger JK, Hiscock J, Neal RD, et al. 'Carrying on the way we are, is becoming shambolic'-an interview study with prostate cancer specialists about their usual practice of follow-up. J Clin Urol 2015;8:240-5.

16. National Institute for Health and Care Excellence. Prostate cancer: diagnosis and treatment-full guideline. Cardiff: National Collaborating Centre for Clinical Excellence, 2008. http://www.nice.org.uk/nicemedia/ live/11924/39687/39687.pdf (accessed 5 Feb 2015).

17. Johansson B, Holmberg L, Berglund G, et al. Reduced utilisation of specialist care among elderly cancer patients: a randomised study of a primary healthcare intervention. Eur J Cancer 2001;37:2161-6128.

18. Nielsen JD, Palshof T, Mainz J, et al. Randomised controlled trial of a shared care programme for newly referred cancer patients: bridging the gap between general practice and hospital. Qual Saf Health Care 2003;12:263-72.

19. Fletcher SG, Clark SJ, Overstreet DL, et al. An improved approach to followup care for the urological patient: drop-in group medical appointments. J Urol 2006;176:1122-6.

20. Anderson $B$. The benefits to nurse-led telephone follow-up for prostate cancer. Br J Nurs 2010;19:1085-90.

21. Jefford M, Aranda S, Gough K, et al. Evaluating a nurse-led survivorship care package (SurvivorCare) for bowel cancer survivors: study protocol for a randomized controlled trial. Trials 2013;14:260.

22. Richardson A, Griffin M, Miller C, et al. Living life after cancer treatment: a nurse-led support service. Cancer Nurs Pract 2008;7:36-8.
23. Ashley L, Jones H, Forman D, et al. Feasibility test of a UK-scalable electronic system for regular collection of patient-reported outcome measures and linkage with clinical cancer registry data: The electronic Patient-reported Outcomes from Cancer Survivors (ePOCS) system. BMC Med Inform Decis Mak 2011;11:66.

24. Holzner B, Giesinger JM, Pinggera J, et al. The Computer-based Health Evaluation Software (CHES): a software for electronic patient-reported outcome monitoring. BMC Med Inform Decis Mak 2012;12:126.

25. Vickers AJ, Savage CJ, Shouery M, et al. Validation study of a webbased assessment of functional recovery after radical prostatectomy. Health Qual Life Outcomes 2010;8:82.

26. Skolarus TA, Wittmann D, Northouse $L$, et al. Recommendations for prostate cancer survivorship care: an update to the 2009 Michigan cancer consortium guidelines for the primary care management of prostate cancer post-treatment sequelae. J Mens Health 2014;11:95-107.

27. Renders CM, Valk GD, Griffin SJ, et al. Interventions to improve the management of diabetes mellitus in primary care, outpatient and community settings. Cochrane Database Syst Rev 2000;(1):CD001481.

28. Gilbody S. Review: disease management programmes improve detection and care of people with depression. Evid Based Mental Health 2004;7:80.

29. Lewis RA, Neal RD, Williams $\mathrm{NH}$, et al. Nurse-led vs conventional physician-led follow-up for patients with cancer: systematic review. $J$ Adv Nurs 2009;65:706-23.

30. Lewis RA, Neal RD, Williams $\mathrm{NH}$, et al. Follow up of cancer in primary care vs secondary care: systematic review. Br J Gen Pract 2009;59:e234-47.

31. O'Brien R, Rose PW, Campbell C, et al. Experiences of follow-up after treatment in patients with prostate cancer: a qualitative study. BJU Int 2010;106:998-1003.

32. Abrahamson K, Durham M, Fox R. Managing the unmet psychosocial and information needs of patients with cancer. Patient Intell 2010;2:45-52.

33. Cockle-Hearne J, Faithfull S. Self-management for men surviving prostate cancer: a review of behavioural and psychosocial interventions to understand what strategies can work, for whom and in what circumstances. Psychooncology 2010;19:909-22.

34. National Institute for Health and Care Excellence. Guidance on cancer services improving supportive and palliative care for adults with cancer-the manual. London: National Institute for Clinical Excellence, 2004. https://www.nice.org.uk/guidance/csgsp/evidence/ supportive-and-palliative-care-the-manual-2 (accessed 5 Feb 2015).

35. Improving outcomes: a strategy for cancer. Department of Health, 2011. https://www.gov.uk/government/uploads/system/uploads/ attachment_data/file/213785/dh_123394.pdf (accessed 20 Feb 2015).

36. Together for Health, Cancer Delivery Plan for the NHS to 2016. NHS Wales, 2012. http://wales.gov.uk/topics/health/publications/ health/strategies/cancer/?lang=en (accessed 3 Nov 2014).

37. Aylward M, Phillips C, Howson H. Simply prudent healthcareachieving better care and value for money in Wales. Discussion paper. http://www.1000livesplus.wales.nhs.uk/sitesplus/documents/ 1011/Bevan\%20Commission\%20Simply\%20Prudent\%20Healthcare \%20v1\%2004122013.pdf (accessed 5 Feb 2015).

38. Watson E, Rose P, Frith E, et al. PROSPECTIV-a pilot trial of a nurse-led psychoeducational intervention delivered in primary care to prostate cancer survivors: study protocol for a randomised controlled trial. BMJ Open 2014;4:e005186.

39. Craig $P$, Dieppe $P$, Macinture $S$, et al. Developing and evaluating complex interventions: The new Medical Research Council guidance. Int J Nurs Stud 2013;50:587-92.

40. Russell D, Hoare ZS, Whitaker R, et al. Generalized method for adaptive randomization in clinical trials. Stat Med 2011;30:922-34.

41. Cancer survival trends in Wales 1985-2004. Cardiff: Welsh Cancer Intelligence and Surveillance Unit (WCISU), 2010. http://www.wcisu. wales.nhs.uk/opendoc/226106 (accessed 20 Feb 2015).

42. Macmillan Cancer Support. Macmillan organiser. 2012. http://be macmillan.org.uk/be/s-508-macmillan-organiser.aspx (accessed 5 Feb 2015).

43. The National Cancer Survivorship Initiative. The detection and management of psychological distress in people with cancer and their families. 2013. http://www.ncsi.org.uk/wp-content/uploads/ course-outline-2013PDF.pdf (accessed 5 Feb 2015).

44. The Christie. Maguire advanced communication Skills. 2014. http:// www.christie.nhs.uk/school-of-oncology/maguire-communicationskills.aspx (accessed 3 Nov 2014).

45. Macmillan Cancer Support. Motivational interviewing. 2013a. http:// learnzone.org.uk/macprofs/course.php?id=12 (accessed 5 Feb 2015). 
46. Macmillan Cancer Support. 10-minute CBT. 2013. http://learnzone. org.uk/macprofs/course.php?id=16 (accessed 5 Feb 2015).

47. National Cancer Survivorship Initiative. Holistic needs assessment for people with cancer-a practical guide for healthcare professionals. 2012. http://www.ncsi.org.uk/wp-content/uploads/Holistic-NeedsAssessment-practical-guide.pdf (accessed 5 Feb 2015).

48. NHS Wales. Canisc. http://www.wales.nhs.uk/nwis/page/52601 (accessed 3 Nov 2014).

49. Macmillan Cancer Support. Assessment and care planning for people with cancer. 2012. http://be.macmillan.org.uk/Downloads/ Cancerlnformation/LivingWithAndAfterCancer/MAC12957assmtcare E02lowrespdf20131121.pdf (accessed 5 Feb 2015)

50. Moorey S, Greer S. Oxford guide to CBT for people with cancer. Oxford, UK: Oxford University Press; 2011:90-102.

51. Watson M, Kissane DW. Handbook of psychotherapy in cancer care. Chichester, UK: Wiley-Blackwell, 2011:15-38.

52. Bandura A. Self-efficacy: toward a unifying theory of behavioral change. Psychol Rev 1977;84:191-215.

53. Szymanski KM, Wei JT, Dunn RL, et al. Development and validation of an abbreviated version of the expanded prostate cancer index composite instrument for measuring health-related quality of life among prostate cancer survivors. Urology 2010;76:1245-50.

54. Zigmond AS, Snaith RP. The hospital anxiety and depression scale. Acta Psychiatr Scand 1983;67:361-70.
55. Lorig KR, Sobel DS, Ritter PL, et al. Effect of a self-management program on patients with chronic disease. Eff Clin Pract 2001;4:256-62.

56. Boyes A, Girgis A, Lecathelinais C. Brief assessment of adult cance patients' perceived needs: development and validation of the 34-item Supportive Care Needs Survey (SCNS-SF34). J Eval Clin Pract 2009;15:602-6.

57. Schofield P, Gough K, Lotfi-Jam K, et al. Validation of the Supportive Care Needs Survey-short form 34 with a simplified response format in men with prostate cancer. Psychooncology 2012;21:1107-12.

58. Brooks R. EuroQol: the current state of play. Health Policy 1996;37:53-72.

59. Ritchie J, Spencer L. Analysing qualitative data. London: Routledge, 1994:3.

60. Ritchie J, Spencer L, O'Connor W. Carrying out qualitative analysis. In: Lewis J, Ritchie J, eds. Qualitative research practice. London: Sage, 2003:219-62.

61. Edwards RT, Hounsome B, Russell D, et al. QALY calculation alongside randomised controlled trials: from the torch to the traffic light. 2004. http://www.ces-asso.org/sites/default/files/H5-Edwards. pdf (accessed 5 Feb 2015).

62. Curtis L. Unit costs of health and social care 2014. Kent: Personal Social Services Research Unit, 2014. http://www.pssru.ac.uk/projectpages/unit-costs/2014/ (accessed 2 Mar 2015). 